\title{
Rinoseptoplastía: Experiencia de 3 años en la Pontificia Universidad Católica de Chile

\author{
Rhinoseptoplasty: Three-year experience
} at the Pontificia Universidad Católica de Chile
}

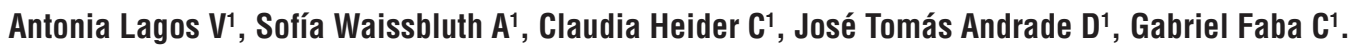

\begin{abstract}
RESUMEN
Introducción: La rinoseptoplastía es una cirugía compleja y desafiante. Permite resolver problemas estéticos y funcionales de la nariz.

objetivo: Describir la experiencia en rinoseptoplastías realizadas en el Comité de Nariz en nuestro centro.

Material y método: Estudio descriptivo de pacientes sometidos a rinoseptoplastía entre 2013 y 2015.

Resultados: Se operaron 265 pacientes en 3 años, 164 (61,8\%) fueron mujeres y la edad promedio fue de 29 años (rango 13-73). Las cirugías fueron principalmente primarias (secundarias: 2,6\%). El abordaje más utilizado fue abierto (92,5\%). Se realizó trabajo de punta nasal en 253 (95,5\%) pacientes; resección del dorso osteocartilaginoso en 252 (95,1\%), y aumento de éste en sólo 5 (1,9\%). Las osteotomías fueron frecuentes: paramedianas en 229 (86,4\%), percutánea lateral bilateral en 217 (81,9\%) y percutánea lateral unilateral en $17(6,4 \%)$. El vástago columelar fue el injerto de punta más frecuentemente utilizado, en 241 (90,9\%) pacientes, seguido del escudo en 69 (26,0\%). En el dorso, se utilizaron autoespaciadores en 124 (46,7\%) pacientes, y espaciadores en 109 (41,1\%).

Conclusiones: El abordaje abierto fue el más frecuente, demostrando ser favorable en nuestro centro por la exposición y aprendizaje de nuestros residentes. Se constató $2,6 \%$ de cirugías secundarias. Se destaca el uso de vástago columelar para otorgar soporte a la punta nasal dada la alta prevalencia de nariz hispana en nuestro centro.
\end{abstract}

Palabras clave: Rinoseptoplastía, punta nasal, dorso, injertos.

\begin{abstract}
Introduction: Rhinoseptoplasty is a complex and challenging surgery. It addresses nasal aesthetics and functionality.

Aim: To describe the experience in rhinoseptoplasty performed by the Nose Committee at our medical center.
\end{abstract}

1 Médico, Departamento Otorrinolaringología, Pontificia Universidad Católica de Chile. 
Materials and methods: Descriptive study, of the all rhinoseptoplasties performed by the Nose Committee between 2013 and 2015.

Results: 265 patients underwent surgery in the 3-year period; 164 (61.8\%) were women and the mean age was 29 years (range 13-73). Most were primary surgeries (secondary: 2.6\%). An external approach was most commonly used (92.5\%). Nasal tip surgery was performed in 253 (95.5\%) patients; osteocartilaginous dorsum reduction in 252 (95,1\%), and augmentation in only 5 (1,9\%). Osteotomies were frequent: paramedian in 229 (86.4\%), bilateral percutaneous lateral osteotomies in 217 (81.9\%) and unilateral percutaneous lateral in 17 (6.4\%). Regarding nasal tip grafts, columellar strut was most often used, in 241 (90,9\%) patients, followed by the shield graft in 69 (26,0\%). For the dorsum, autospreaders were used in 124 (46.7\%), and spreader grafts in 109 (41.1\%).

Conclusions: Open rhinoseptoplasty was the most commonly used approach. This has shown favorable for residents due to better exposition of nasal anatomy. Only 2.6\% were secondary rhinoseptoplasties. We emphasize the use of the columellar strut for nasal tip support as we frequently encounter hispanic noses at our center.

Key words: Rhinoseptoplasty, nasal tip, dorsum, grafts.

\section{INTRODUCCIÓN}

La rinoseptoplastía es una cirugía compleja y desafiante debido a que se basa en un objetivo funcional y estético, requiriendo adaptarse a las necesidades de cada paciente en forma individualizada. Existen múltiples técnicas descritas y una constante evolución de las mismas durante los últimos años.

El abordaje quirúrgico puede ser abierto 0 cerrado, dependiendo de la experiencia y/o preferencia del cirujano y del tipo de alteraciones que se desea modificar. Se ha demostrado que ambos abordajes generan resultados estéticos y funcionales adecuados. El abordaje abierto tiene la ventaja de permitir una excelente visualización y acceso directo a las estructuras nasales, pero tiene los inconvenientes de cursar con mayor edema posoperatorio y de poder presentar una cicatriz en la columela en la mayoría imperceptible a los 6 meses $^{1,2}$. El abordaje cerrado puede ser eficiente en una cirugía que requiera cambios mínimos, 0 en una nariz que no ha sido previamente traumatizada, con ausencia de patologías congénitas, o para una nariz más bien simétrica ${ }^{3}$.

En Chile, es frecuente de ver una nariz hispana, también conocida como latina o mestiza. Es importante realizar un diagnóstico anatómico adecuado ya que este tipo de nariz suele presentar una piel gruesa con tejidos subyacentes débiles. Los huesos propios tienden a ser cortos y los cartílagos delgados y débiles. La punta nasal tiende a ser bulbosa, poco definida, con hipoproyección e hiporrotación ${ }^{4}$. Tomando esto en cuenta, los motivos de consulta frecuentes en relación a lo estético son obtener una punta nasal más proyectada, rotada y definida. El uso de injertos, cirugías más conservadoras y uso de suturas permite lograr un resultado estético adecuado.

Cuando estos pacientes acuden al otorrinolaringólogo, consultan frecuentemente por obstrucción nasal. El examen clínico es fundamental para determinar el nivel de la obstrucción que puede corresponder a una desviación septal, hipertrofia de los cornetes inferiores $y / 0$ compromiso de la válvula nasal interna y/0 externa ${ }^{5}$. Si, por ejemplo, el paciente presenta una desviación septal sintomática, se debe considerar una septoplastía la cual permite alinear el tabique y obtener cartílago para los injertos 6 .

Según el motivo de consulta de los pacientes, se puede optar por una rinoplastía con o sin septoplastía. Las rinoplastías han demostrado mejorar la autoestima y la calidad de vida de los pacientes. Se sienten más atractivos, perciben ser más exitosos y estar en mejor estado de salud ${ }^{7,8}$. En el Comité de Nariz de nuestro centro, se realizan principalmente rinoseptoplastías debido a que la mayoría de las consultas son por causas estéticas y funcionales. 


\section{OBJETIVO}

El objetivo del estudio es describir la experiencia en rinoseptoplastías indicadas por el Comité de Nariz del Departamento de Otorrinolaringología de la Pontificia Universidad Católica de Chile.

\section{MATERIAL Y MÉTODO}

Se realizó un estudio descriptivo donde se analizaron los protocolos quirúrgicos de todos los pacientes sometidos a una rinoplastía con o sin septoplastía entre los años 2013 y 2015 en el Comité de Nariz del Departamento de Otorrinolaringología de la Pontificia Universidad Católica de Chile. Este consta de un equipo formado por un otorrinolaringólogo experto en rinoplastías junto con un residente de tercer año de la especialidad. Se registraron y analizaron características biodemográficas como el abordaje utilizado, las técnicas quirúrgicas empleadas para cada paciente en relación a la punta nasal, el dorso osteocartilaginoso, las osteotomías realizadas y los injertos utilizados. Se analizaron los resultados mediante estadística descriptiva, con medidas de tendencia central y rango. Este estudio fue aprobado por el Comité de Ética de nuestro centro hospitalario.

\section{RESULTADOS}

Dentro de un período de tres años, se operaron 265 pacientes en el Comité de Nariz. Predominó el sexo femenino, siendo 164 mujeres $(61,9 \%$ ) y la edad promedio fue de 29 años (rango 13-73 años). La cantidad de cirugías no varió de forma importante por año. Se realizaron 87 cirugías en el año 2013, 80 cirugías en el año 2014, y 98 cirugías en el año 2015; promediando 88 cirugías por año. De los 265 pacientes, $263(99,2 \%)$ correspondieron a rinoseptoplastías, siendo sólo 2 (0,7\%) únicamente rinoplastías. El abordaje abierto fue el más utilizado, en 245 (92,5\%) casos. La mayoría de las cirugías correspondieron a cirugías primarias, siendo sólo siete $(2,6 \%)$ cirugías secundarias.

La mayoría de los pacientes requirió trabajo de punta nasal, el cual fue necesario en 253 casos $(95,5 \%)$. Lo más frecuente fue la realización de puntos intercruras mediales y medias a 6 y $2 \mathrm{~mm}$ del domo respectivamente (también conocido como "triángulo de Sheen") en 241 (90,9\%) pacientes. Luego se encontró la plicatura de domos y la resección cefálica de la crura lateral (Figura 1). Menos frecuentes fueron el punto septocolumelar y punto de "Tebbets" o punto intercruras laterales (Tabla 1).

En cuanto al manejo de dorso, éste fue necesario en $257(97,0 \%)$ casos, la mayoría corres-

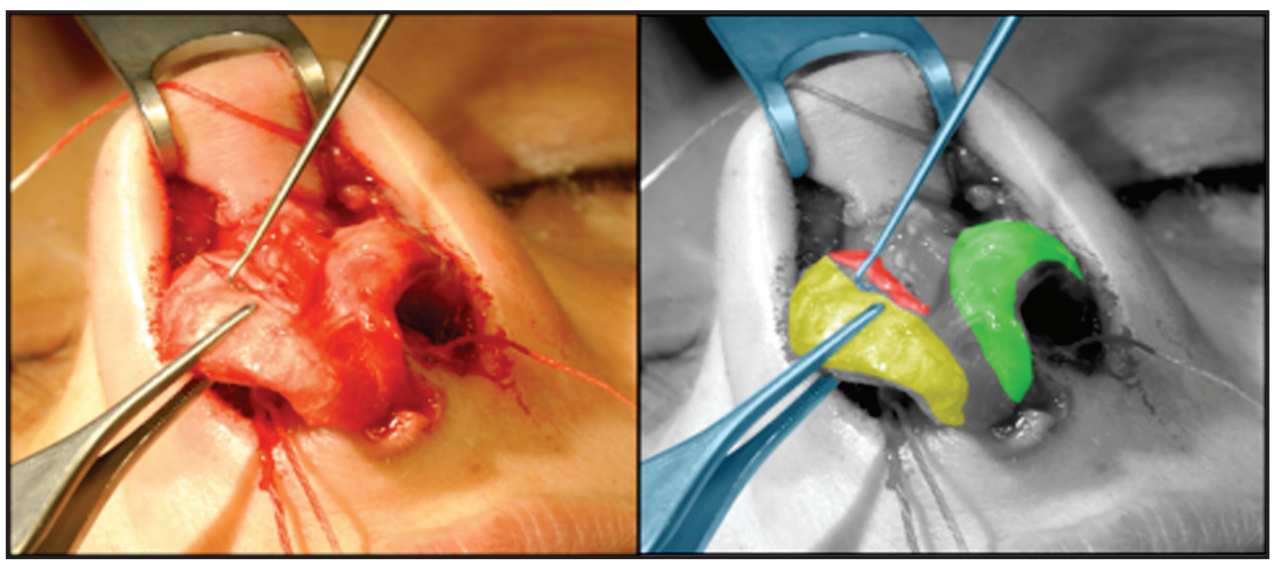

Figura 1. Exposición por abordaje abierto: resección cefálica de la crura lateral del cartílago alar. (A) Abordaje abierto, exponiendo cartílagos alares. (B) Resección cefálica (rojo) de la crura lateral derecha (amarillo). 
Tabla 1. Variedad de técnicas utilizadas para el manejo de la punta nasal

\begin{tabular}{|lcc|}
\hline Técnica & Número de pacientes & Porcentaje (\%) de total de pacientes n=265 \\
\hline Total de trabajo de punta nasal & 253 & 95,5 \\
"Triángulo de Sheen" & 241 & 90,9 \\
Plicatura de domos & 237 & 89,4 \\
Resección cefálica crura lateral & 220 & 83,0 \\
Punto septocolumelar & 154 & 58,1 \\
Punto de Tebbets & 48 & 18,1 \\
Otro & 10 & 3,8 \\
\hline
\end{tabular}

"Triángulo de Sheen": puntos intercruras mediales y medias a 6 y 2 mm del domo; Punto de Tebbets: punto intercuras laterales.

pondiendo a resección $(95,1 \%)$. Solo $5(1,9 \%)$ pacientes requirieron aumento de dorso, de los cuales uno correspondió a cirugía secundaria. Respecto a las osteotomías, la mayoría de los casos requirió osteotomías paramedianas y laterales bilaterales, en $229(86,4 \%)$ y $217(81,9 \%)$ pacientes respectivamente. Solo $17(6,4 \%)$ pacientes requirieron osteotomías laterales unilaterales. Todas las osteotomías laterales fueron externas percutáneas.

Se utilizaron injertos en $261(98,5 \%)$ pacientes, tanto para la punta nasal como el dorso. El origen del injerto fue principalmente cartílago septal $(93,9 \%)$ y en ocasiones de cartílago auricular $(2,3 \%)$. Se utilizaron injertos de punta nasal en 246 pacientes, correspondiente a $92,8 \%$ del total (Tabla 2). El más frecuente fue el vástago columelar, y en menor frecuencia el escudo, el listón alar, injerto en paraguas y refuerzo alar. En cuanto al manejo del dorso, lo más utilizado fue el autoespaciador en $124(46,8 \%)$ pacientes (el cual no es un injerto propiamente tal). De los injertos expansores, fueron más frecuentes los bilaterales (Tabla 3 ).

De las siete rinoseptoplastías secundarias que se realizaron, cinco consultaron por motivos estéticos y funcionales y dos por interés únicamente estético. De ellos, seis fueron por abordaje abierto, requiriendo manejo de punta nasal con uso de injertos. Se utilizaron espaciadores o autoespaciadores en tres pacientes. Solo una cirugía fue por abordaje cerrado, en la cual se realizó resección de dorso sin requerir trabajo de punta ni injertos de dorso o punta. Los injertos fueron obtenidos

Tabla 2. Injertos utilizados para el trabajo de la punta nasal

\begin{tabular}{|lcc|}
\hline Tipo de injerto & Número de pacientes & Porcentaje (\%) de total de pacientes n=265 \\
\hline Uso de injerto & 246 & 92,8 \\
Vástago columelar & 241 & 90,9 \\
Escudo & 69 & 26,0 \\
Listón alar & 4 & 1,5 \\
En paraguas & 1 & 0,3 \\
Reborde alar & 1 & 0,3 \\
\hline
\end{tabular}

Tabla 3. Injertos utilizados para el manejo del dorso nasal

\begin{tabular}{|lcc|}
\hline Tipo de injerto & Número de pacientes & Porcentaje de total de pacientes $n=265$ \\
\hline Autoespaciador $^{*}$ & 124 & 46,8 \\
Espaciador unilateral & 88 & 33,2 \\
Espaciador bilateral & 21 & 7,9 \\
\hline
\end{tabular}

${ }^{*}$ No es un injerto propiamente tal. 
del cartílago auricular en mayor porcentaje que en las cirugías primarias, correspondiendo al $40 \%$ de los pacientes.

\section{DISCUSIÓN}

La rinoseptoplastía es una cirugía frecuente pero desafiante, ya que se debe adaptar a las necesidades funcionales y estéticas del paciente. En nuestro centro la mayoría de las cirugías cumplen funciones estéticas y funcionales. En base a este estudio podemos ver que se utiliza más frecuentemente el abordaje abierto y predomina el trabajo de punta con uso de injertos, específicamente el vástago columelar.

El abordaje utilizado puede variar según la experiencia $y / 0$ preferencia del cirujano, siempre adaptándose a las necesidades individuales de cada paciente. En nuestro centro se prefiere el abordaje abierto, ya que permite una mejor exposición de la anatomía nasal, facilita el trabajo de la punta nasal y genera una curva de aprendizaje más rápida en nuestros residentes. Destaca el alto porcentaje de necesidad de trabajo de punta nasal, correspondiente al 95,5\% de los casos, lo cual podría deberse a la alta prevalencia de nariz hispana en Chile. Los hallazgos anatómicos que se buscan corregir en este fenotipo nasal son los cartílagos laterales inferiores anchos, crura lateral cóncava 0 convexa, hipoproyección e hiporrotación de la punta nasal, distancia interdomal aumentada, y punta nasal bulbosa y mal definida 4 . Esto podría explicar el alto porcentaje de realización del "triángulo de Sheen" y plicatura de domos, para lograr una mejor definición de la punta nasal, reducir la distancia interdomal y mejorar la proyección y rotación de la punta nasal. La resección cefálica de la crura lateral del cartílago lateral inferior, también muy utilizada en nuestro centro, permite corregir la mal posición cefálica de éstas. La tendencia es siempre a conservar más que resecar las estructuras nasales. La resección cefálica es conservadora, dejando al menos $8 \mathrm{~mm}$ de crura lateral, y se acompaña de técnicas de sutura para lograr un resultado estético adecuado sin riesgo de colapso ni retracción de la pared nasal lateral a largo plazo ${ }^{9}$.

La nariz hispana además se caracteriza por presentar cartílagos laterales superiores débiles, hue- sos propios cortos y tercio medio estrecho. Esto se puede trabajar utilizando injertos de soporte, como son los espaciadores y/o autoespaciadores que permiten dar soporte a los cartílagos laterales, prevenir el colapso valvular y evitar la aparición de "V invertida"4. La importancia de estos injertos se ve reflejada en la alta frecuencia con que fueron utilizados en nuestro estudio. Sólo en $26 \%$ no fue necesario realizar autoespaciadores ni colocar injertos espaciadores.

Los injertos de punta nasal también contribuyen en el manejo de las alteraciones anatómicas mencionadas. El vástago columelar fue el injerto de punta más utilizado en nuestro estudio $(90,9 \%)$, que corresponde a un injerto de cartílago rectangular angosto pero firme que se posiciona entre ambas cruras mediales de los cartílagos laterales inferiores y luego se fija con suturas. Este permite dar mayor soporte, rotación y proyección a la punta. El escudo fue el segundo injerto de punta nasal más utilizado $(26 \%)$ y corresponde a un injerto trapezoidal de cartílago preferentemente septal que se posiciona sobre las cruras mediales en línea media, logrando una mejor definición de la punta ${ }^{4}$. Resultados similares han sido reportados en Chile donde destaca el uso del vástago como injerto predominante para la punta nasal. Se ha descrito el uso del vástago en $93 \%$ de los pacientes operados de rinoplastía primaria en pacientes chilenos ${ }^{10}$.

En las rinoplastías estéticas se ha descrito como complicación frecuente la obstrucción nasal, presente entre $15 \%$ a $68 \%$ de los pacientes operados, siendo la principal indicación de cirugía secundaria ${ }^{11-13}$. Las rinoseptoplastías secundarias son necesarias en $5 \%$ a $15 \%$ de los $\operatorname{casos}^{12,13}$, incidencia levemente mayor a la encontrada en nuestro estudio, de 2,6\%. Esto podría deberse a que los pacientes con segunda consulta, acuden a otros centros para la resolución definitiva en caso de disconformidad.

\section{CONCLUSIÓN}

Existen diversas técnicas para la rinoseptoplastía, las cuales se encuentran en constante evolución y son utilizadas según la experiencia del cirujano y necesidades del paciente. El abordaje abierto fue el más utilizado en nuestro estudio. Este ha mostrado 
resultados favorables tanto para el paciente como para los residentes por la mayor exposición y visualización. La tasa de rinoseptoplastías secundarias fue de 2,6\%.

\section{BIBLIOGRAFÍA}

1. Zijlker T, Adamson P. Open StRUCTURE RHINOPLASTY. Clin Ololaryngol 1993; 18: 125-34.

2. Perkins $S$. The evolution of the combined use of endonasal and external columellar approaches to rhinoplasty. Facial Plast Surg Clin N Am 2004; 12: $35-50$.

3. Fedok F. Primary Rhinoplasty. Facial Plast Surg Clin North Am 2016; 24: 323-35.

4. Сово R. Rhinoplasty in Latino Patients. Clin Plast Surg 2016; 43: 237-54.

5. Michel J, Achache M, Thomassin J, Dessi P. Ventilatory examination before an aesthetic rhinoplasty. Ann Chir Plast Esthet 2014; 59 : 392-9.

6. Bussi M, Palonta F, Toma S. Grafting in revision rhinoplasty. Acta Otorhinolaryngologica Italica 2013; 33: 183-89.

7. Nellis J, Ishil M, Bater K, Papel I, Kontis T, Byrne P, Et AL. Association of Rhinoplasty With Perceived
Se destaca el uso de vástago columelar para otorgar soporte a la punta nasal dada la alta prevalencia de nariz hispana en nuestro centro.

Attractiveness, Success, and Overall Health. JAMA Facial Plast Surg 2017 Oct 19.

8. Niehaus R, Kovacs L, Machens HG, Herschbach P, Papadopulos NA. Quality of Life-Changes after Rhinoplasty. Facial Plast Surg 2017; 33: 530-36.

9. Сово R. Rhinoplasty in the Mestizo nose. Facial Plast Surg Clin North Am 2014; 22: 395-415.

10. Casanueva F. Injertos de punta nasal en rinoplastía primaria. Rev Otorrinolaringol Cir Cabeza Cuello 2016; 76: 21-30.

11. FodA HM. Rhinoplasty for the multiply revised nose. Am J Otolaryngol 2005; 26(1): 28-34.

12. Chauhan N, Alexander AJ, Sepehr A, Adamson P. Patient complaints with primary versus revision rhinoplasty: analysis and practice implications. Aesthetic Surg J 2011; 31: 775-80.

13. Yu K, Kim A, Pearlman SJ. Functional and aesthetic concerns of patients seeking revision rhinoplasty. Arch Facial Plast Surg 2010; 12: 291-7.

Dirección: José Tomás Andrade Donoso Marcoleta 350, Centro de Especialidades Médicas, Hospital Clínico Pontificia Universidad Católica de Chile, Santiago E mail: tomasandrades@gmail.com 